\title{
Acute thrombotic occlusion of the superior vena cava treated successfully by emergency thrombectomy
}

\author{
V. KETHARANATHAN, IAN MCCONCHIE, a nd \\ MICHAEL MULLERWORTH
}

Cardio-thoracic Surgery Unit, The Royal Melbourne Hospital

\begin{abstract}
We report the first recorded case of acute thrombotic occlusion of the superior vena cava successfully treated by disobliterative thrombectomy. The caval thrombosis was caused by the infusion into the superior vena cava, over a period of six days, of fluids, electrolytes, aminoacids, concentrated carbohydrates, and antibiotics. Over a period of four hours, the patient became moribund due to the development of the 'wet brain syndrome'. Disobliterative caval thrombectomy, performed as an emergency procedure, resulted in immediate and permanent relief.
\end{abstract}

\section{CASE HISTORY}

Mrs. M. T., a 56-year-old housewife, was admitted to the Royal Melbourne Hospital on 7 August 1969. She was suffering from achalasia of the cardia, megaoesophagus, and a complicating fistula between the oesophagus and the lower lobe of the right lung which caused deteriorating dysphagia, severe malnutrition, and aspiration pneumonia. Increasingly frequent attacks of aspiration pneumonia and progressive weight loss had troubled her for 25 years. Oesophagoscopy and biopsy before admission to hospital showed that the oesophagopulmonary fistula was not the result of carcinoma in the megaoesophagus.

Partial oesophagogastrectomy and closure of the pulmonary end of the oesophagopulmonary fistula, through abdominal and right thoracotomy incisions, was planned. Dehydration, electrolyte inbalance, anaemia, malnutrition, and lung infection had to be corrected before operation. Therefore, on 7 August 1969 a plastic cannula was inserted into the superior vena cava through the left subclavian vein. Through this, fluid, electrolytes, aminoacids, concentrated carbohydrates, and antibiotics were administered. The proposed operation was performed on 12 August 1969. A tracheostomy was performed at the end of the operation and intermittent positive pressure respiration was used postoperatively.

On the morning after operation the patient seemed well, but central venous pressure could not be recorded through the caval cannula although fluids could be infused slowly. Consequently, a second plastic cannula was inserted into the superior vena cava through the right external jugular vein. A portable chest radiograph showed that the two cannulae were at different levels in the superior vena cava. Fluid infusion and central venous pressure measurements through the external jugular cannula were satisfactory.
At 2.00 p.m. that afternoon, 24 hours after the end of the operation, the patient's condition suddenly deteriorated gravely. She became dyspnoeic, deeply cyanosed, and then unconscious. The neck and scalp veins were tensely distended. She had tachycardia of 160 per minute, arterial hypotension of $110 / 70$ $\mathrm{mmHg}$, and central venous hypertension of $40 \mathrm{mmHg}$. It was thought that she had a major pulmonary embolus; but neither electrocardiogram, chest radiograph, nor examination of the lower limbs supported that diagnosis. However, heparin and isoprenaline were administered intravenously and her conscious state, cyanosis, arterial hypotension, and venous hypertension improved a little.

Two hours later, at 4.00 p.m. on 13 August 1969, she relapsed-unconscious, apnoeic, and with even more marked venous hypertension and arterial hypotension.

In spite of lack of confirmatory evidence, it was thought that she probably had had a further major pulmonary embolus and that pulmonary embolectomy offered the only hope of survival.

It was decided to try to confirm the diagnosis of pulmonary embolus by pulmonary arteriogram before embolectomy. At 6.00 p.m. she was moved to the radiography department, unconscious and being artificially ventilated because she was still apnoeic. Injection of Urografin into the superior vena cava, through the left subclavian and right external jugular veins, indicated that the superior vena cava was completely blocked up to the junction of the left and right innominate veins (Figure). Detachment of some superior vena caval thrombus with subsequent pulmonary embolization was regarded as a possibility, so a catheter was passed up into the right atrium through a percutaneous puncture in the left femoral vein and injection of Urografin through that catheter into the right atrium showed the lower limit of the superior vena caval thrombus just above the junction 

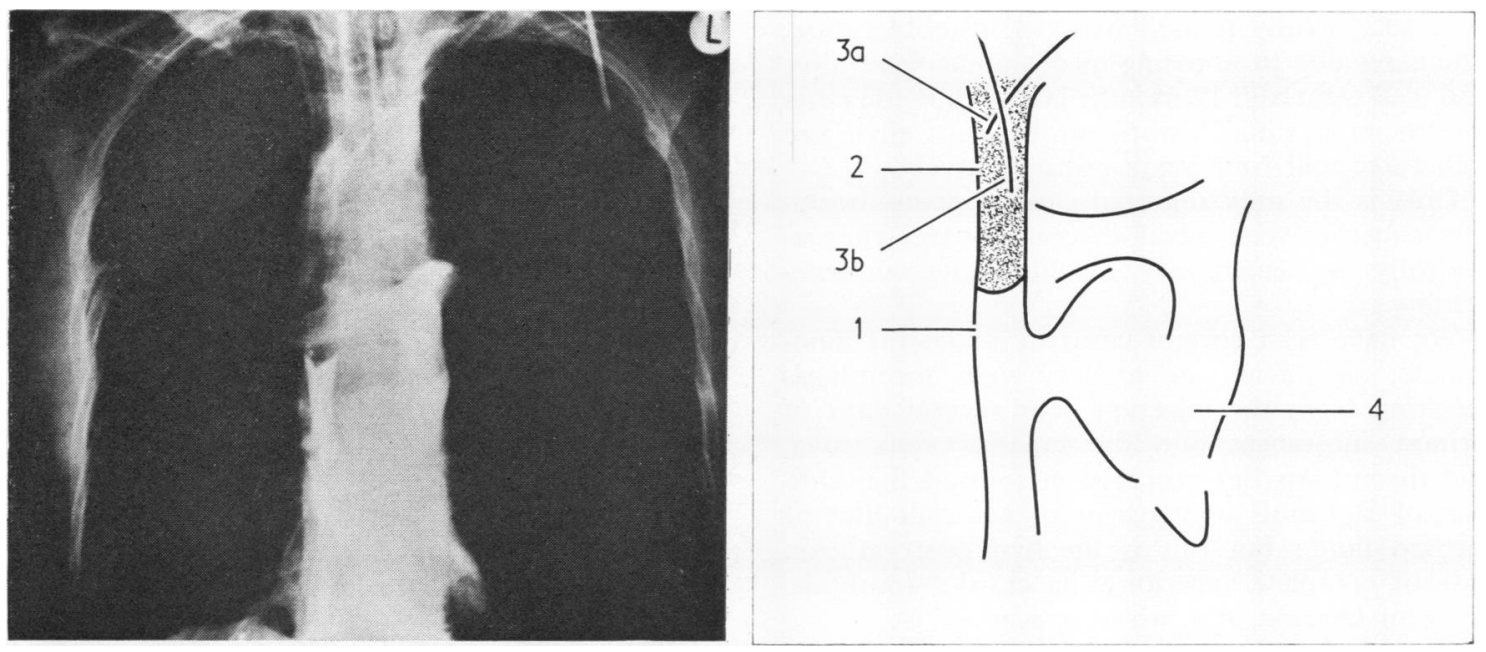

FIGURE. Radiograph showing (1) Urografin-filled intrapericardial superior vena cava; (2) thrombus-filled extrapericardial superior vena cava; (3) catheters in superior vena cava-via left subclavian vein (3a) and right external jugular vein ( $3 b$ ); (4) Urografin in right heart, main pulmonary artery, and left and right pulmonary arteries.

of the superior vena cava and the right atrium. It also showed clearly that there was no major pulmonary artery obstruction. At this stage, for the first time, it was realized that the cyanosis and venous congestion were confined to the upper half of the body, a condition typical of acute superior vena caval obstruction. It was also realized that intravenous infusion, during the four hours after the onset of the caval obstruction, had been aggravating her 'wet brain syndrome'. Obviously, her only hope of survival was immediate disobliterative caval thrombectomy.

The patient was taken from the radiography department to the operating theatre. The right thoracotomy incision was re-opened and inspection confirmed thrombosis of the whole length of the extrapericardial part of the superior vena cava. The pericardium was opened longitudinally anterior to the phrenic nerve and $2.5 \mathrm{~cm}$ of patent intrapericardial superior vena cava was seen. Two slings were placed around the patent intrapericardial superior vena cava and the central control sling was tightened to prevent embolism from the superior vena cava into the right heart. A longitudinal incision was made in the superior vena cava between the slings and much clot was removed from the superior vena cava by an open-ended sucker and a Fogarty venous catheter. After thrombectomy, bleeding from the peripheral end was free and forceful. A tangential clamp was placed deep to the caval incision and the vena cava was repaired with a 5 "O" Polydec suture. The central venous pressure fell from $40 \mathrm{mmHg}$ to $4 \mathrm{mmHg}$ and the patient regained consciousness as soon as the anaesthetic was discontinued. Subsequent recovery was quite uneventful.
Microscopic examination of the thrombus showed it to be recent.

The patient was last seen in December 1970 when she reported that she was eating normal food without blockage and without vomiting. She had gained $11 \mathrm{~kg}$ in weight. She had not had any further attacks of pneumonia. On examination there was no evidence of recurrent superior vena caval obstruction.

\section{DISCUSSION}

Replacement or bypass of the chronically occluded superior vena cava is sometimes necessary, and Gerbode, Yee, and Rundle (1949) were the first to report such a procedure using endogenous and exogenous grafts.

Disobliteration of six chronically occluded superior venae cavae has been reported in five papers (Blondeau, Wapler, Piwnica, and Dubost, 1959 ; Hanlon and Danis, 1965 ; Pate and Hammon, 1965; Templeton, 1962; O'Neill, 1954): Two cases were due to malignant intracaval occlusion and operation resulted in improvement. However, Dubost's patient died of recurrence of sarcoma one year later and Templeton's patient died of recurrent malignancy one year and six weeks later. Four cases of chronic caval thrombosis due to benign conditions have been reported (Hanlon and Danis, 1965; Pate and Hammon, 1965; Templeton, 1962; O'Neill, 1954). Two were due to fibrosing mediastinitis, and, in the other two, the underlying pathology was histoplasmosis. All 
were successfully treated by caval disobliteration. The cases due to fibrosing mediastinitis were alive and well eight and 12 months later, while the cases due to mediastinal histoplasmosis were alive and well three and four years postoperatively.

Ours is the only reported case of acute, occlusive superior vena caval thrombosis treated successfully by emergency disobliterative thrombectomy.

We have seen several cases of unilateral innominate, subclavian or axillary vein thrombosis resulting from the injection over several days of irritant substances into the superior vena cava, and thrombosis has occurred in spite of the addition of 500 units of heparin to each half litre of infused fluid; but this is the first post infusion case of complete superior vena caval thrombosis, acute or chronic, that we have seen.

Had we thought of the possibility of acute caval thrombosis, we would have discarded the original diagnosis of pulmonary embolism. When we examined her legs the only significant observation was the absence of evidence of deep vein thrombosis. The four-hour delay almost cost this patient her life.

\section{REFERENCES}

Blondeau, P., Wapler, C., Piwnica, A., and Dubost, C. (1959). Deux cas de syndrome de la veine cave supérieure traités chirurgicalement avec succès l'un par désobstruction, l'autre par greffe. Arch. Mal. Coeur, 52, 504.

Gerbode, F., Yee, J., and Rundle, F. F. (1949). Experimental anastomoses of vessels to the heart. Possible application to superior vena caval obstruction. Surgery, 25, 556.

Hanlon, C. R., and Danis, R. K. (1965). Superior vena caval obstruction: indications for diagnostic thoracotomy. Ann. Surg., 161, 771.

O'Neill, T. J. (1954). In discussion on Scannell, J. G., and Shaw, R. S. Surgical reconstruction of the superior vena cava. J. thorac. Surg., 28, 163.

Pate, J. W., and Hammon, J. (1965). Superior vena cava syndrome due to histoplasmosis in children. Ann. Surg., $161,778$.

Templeton, J. Y. (1962). Endvenectomy for the relief of obstruction of the superior vena cava. Amer. J. Surg., 104, 70. 